InIFolPharm

\title{
Erhaltungstherapie verbessert Gesamtüberleben
}

\author{
Kürzlich präsentierte Daten bestätigen die \\ Erhaltungstherapie als ein Konzept, von dem Patienten \\ mit fortgeschrittenem nicht kleinzelligem Bronchial- \\ karzinom (NSCLC) profitieren können.
}

Viele Patienten mit fortgeschrittenem NSCLC bekommen aufgrund des nach der Primärtherapie verschlechterten Allgemeinzustands keine Zweitlinientherapie mehr. Bisher waren Ergebnisse von Studien mit einer Switch-Erhaltungstherapie, bei der ein anderes Therapeutikum gewählt wird als in der Primärtherapie, auch nicht überzeugend, erklärte Werner Leitmann, Bad Homburg.

Die PARAMOUNT-Studie ist die erste Studie, in der das Ziel einer Verbesserung des Gesamtüberlebens (OS) mit einer Erhaltungstherapie im eigentlichen Sinn erreicht wurde. Im Unterschied zur Switch-Erhaltungstherapie wird die Be- handlung dabei mit einem Wirkstoff fortgesetzt, der bereits Bestandteil der Induktionstherapie war. Laut Christian Schumann, Ulm, erhielten in der PARAMOUNT-Studie 939 chemonaive Patienten mit Nicht-Plattenepithel-NSCLC im Stadium IIIB/IV eine Induktionstherapie mit vier Zyklen Cisplatin/Pemetrexed (Alimta ${ }^{\oplus}$.

Die 539 Patienten, die ein objektives Ansprechen oder eine Stabilisierung erreicht hatten, bekamen anschließend randomisiert eine Erhaltungstherapie mit Pemetrexed oder Placebo, wobei bei allen zusätzlich eine optimale Supportivtherapie vorgesehen war.
Wie Ergebnisse bereits letztes Jahr gezeigt hatten, verlängerte die Erhaltungstherapie mit Pemetrexed das progressionsfreie Überleben (primärer Endpunkt) signifikant auf 4,1 vs. 2,8 Monate ( $\mathrm{p}=$ 0,00006) [Paz-Ares LG et al. J Clin Oncol. 2011;29(Suppl):Abstr. CRA7510].

Nach Daten, die in diesem Jahr vorgestellt wurden, haben Patienten mit Pemetrexed-Erhaltungstherapie auch ein signifikant längeres OS (13,9 vs. 11,0 Monate, p $=0,0196)$ [Paz-Ares LG et al. J Clin Oncol. 2012; 30(Suppl):Abstr. LBA7507]. Schumann betonte dabei: „Alle untersuchten Subgruppen profitierten von der Erhaltungstherapie“.

Günter Springer

Presse-Roundtable „Lilly Post-ASCO Update: Neue Daten zur NSCLC-Erhaltungstherapie mit Pemetrexed" am 4. Juli 2012 in Frankfurt/Main; Veranstalter: Lilly.

\section{Neue Hoffnungsträger bei HER2-Überexpression}

\author{
Das Verständnis der Resistenzmechanismen bei der \\ Behandlung mit monoklonalen Antikörpern gegen den \\ HER2-Rezeptor eröffnet neue Chancen zur Therapie des \\ HER2-überexprimierenden Mammakarzinoms.
}

Patientinnen mit HER2-überexprimierendem metastasiertem Mammakarzinom (mBC) erreichen mit der Kombination von Trastuzumab (T) und einem Taxan ein längeres Gesamtüberleben, aber letztlich ist der Effekt begrenzt. Als eine Ursache der Resistenz gegen T wird die Dimerisierung des HER2-Rezeptors mit HER3 diskutiert, erläuterte Andreas Schneeweiß, Heidelberg. Diese wird durch den monoklonalen Antikörper Pertuzumab (Perjeta ${ }^{\bowtie}$ selektiv unterdrückt.

Die Hypothese, dass sich mit Pertuzumab ein wichtiger Resistenzmechanismus gegen Trastuzumab umgehen lässt, bestätigte sich zunächst in präklinischen Modellen und nun auch in einer PhaseIII-Zulassungsstudie [Baselga J et al. N Engl J Med. 2012;366(2):109-19]. Wie
Schneeweiß berichtete, erhielten $806 \mathrm{~Pa}-$ tientinnen zusätzlich zu einer FirstlineTherapie mit T/Docetaxel entweder Placebo oder Pertuzumab. Obwohl etwa die Hälfte der Frauen bereits eine (neo)adjuvante Chemotherapie bekommen hatte, verlängerte die zusätzliche Gabe von Pertuzumab das progressionsfreie Überleben (PFS) signifikant um 6,1 Monate $(18,5$ vs. 12,4 Monate, $\mathrm{p}<0,0001)$ und die Mortalität verminderte sich signifikant um 36\% ( $\mathrm{p}=0,0053)$.

Einen absoluten Durchbruch in der Krebsmedizin könne das TrastuzumabEmtansine (T-DM1) werden, so Nadja Harbeck, München. T-DM1 besteht aus Trastuzumab und einer hochaktiven Chemotherapie (DM1), die miteinander chemisch verbunden sind. T-DM1 ist im Blut stabil, bindet an den HER2-Rezep- tor im Tumor und wird in die Krebszelle internalisiert. Erst dann erfolgt die Abspaltung des hochaktiven Chemotherapieanteils DM1 von T. Daher ist die systemische Toxizität von T-DM1 gering, betonte Harbeck.

In einer auf dem ASCO 2012 vielbeachteten Phase-III-Studie wurde T-DM1 bei Patientinnen, die unter T/Taxan einen Progress entwickelt hatten, mit dem aktuellen Zweitlinien-Standard (Capecitabin/Lapatinib) verglichen [Blackwell K et al. J Clin Oncol. 2012; 30(suppl):Abstr LBA1]. Die Patientinnen der T-DM1-Gruppe lebten nicht nur signifikant länger progressionsfrei ( $9,6 \mathrm{vs}$. 6,4 Monate, $\mathrm{p}<0,0001)$, sie lebten auch insgesamt länger $(65,4$ vs. $47,5 \%$ nach 2 Jahren, $\mathrm{p}=0,0005)$.

Günter Springer

Pressekonferenz "mHER Zukunft beim HER2-positiven metastasierten Brustkrebs - Neue Wege in der 1st- und 2nd-Line-Therapie" am 5. Juli 2012 in Stuttgart; Veranstalter: Roche. 